\title{
Regression from Dependent Observations
}

\author{
Constantinos Daskalakis \\ EECS \& CSAIL, MIT \\ USA \\ costis@csail.mit.edu
}

\author{
Nishanth Dikkala \\ EECS \& CSAIL, MIT \\ USA \\ nishanthd@csail.mit.edu
}

\author{
Ioannis Panageas \\ ISTD \& SUTD \\ Singapore \\ ioannis@sutd.edu.sg
}

\begin{abstract}
The standard linear and logistic regression models assume that the response variables are independent, but share the same linear relationship to their corresponding vectors of covariates. The assumption that the response variables are independent is, however, too strong. In many applications, these responses are collected on nodes of a network, or some spatial or temporal domain, and are dependent. Examples abound in financial and meteorological applications, and dependencies naturally arise in social networks through peer effects. Regression with dependent responses has thus received a lot of attention in the Statistics and Economics literature, but there are no strong consistency results unless multiple independent samples of the vectors of dependent responses can be collected from these models. We present computationally and statistically efficient methods for linear and logistic regression models when the response variables are dependent on a network. Given one sample from a networked linear or logistic regression model and under mild assumptions, we prove strong consistency results for recovering the vector of coefficients and the strength of the dependencies, recovering the rates of standard regression under independent observations. We use projected gradient descent on the negative log-likelihood, or negative log-pseudolikelihood, and establish their strong convexity and consistency using concentration of measure for dependent random variables.
\end{abstract}

\section{CCS CONCEPTS}

- Mathematics of computing $\rightarrow$ Markov networks; Maximum likelihood estimation; Distribution functions; Multivariate statistics; • Theory of computation $\rightarrow$ Random network models; Sample complexity and generalization bounds.

\section{KEYWORDS}

logistic regression, linear regression, ising models, estimation, gaussian graphical models

\section{ACM Reference Format:}

Constantinos Daskalakis, Nishanth Dikkala, and Ioannis Panageas. 2019. Regression from Dependent Observations. In Proceedings of the 51st Annual ACM SIGACT Symposium on the Theory of Computing (STOC '19), June 23-26, 2019, Phoenix, AZ, USA. ACM, New York, NY, USA, 9 pages. https: //doi.org/10.1145/3313276.3316362

Permission to make digital or hard copies of all or part of this work for personal or classroom use is granted without fee provided that copies are not made or distributed for profit or commercial advantage and that copies bear this notice and the full citation on the first page. Copyrights for components of this work owned by others than the author(s) must be honored. Abstracting with credit is permitted. To copy otherwise, or republish, to post on servers or to redistribute to lists, requires prior specific permission and/or a fee. Request permissions from permissions@acm.org.

STOC '19, fune 23-26, 2019, Phoenix, AZ, USA

(1) 2019 Copyright held by the owner/author(s). Publication rights licensed to ACM. ACM ISBN $978-1-4503-6705-9 / 19 / 06 \ldots \$ 15.00$

https://doi.org/10.1145/3313276.3316362

\section{INTRODUCTION}

Linear and logistic regression are perhaps the two most prominent models in Statistics. In their most standard form, these models postulate that a collection of response variables $y_{1}, \ldots, y_{n}$, which are scalar and binary respectively, are linearly related to a collection of covariates $\mathbf{x}_{1}, \ldots, \mathbf{x}_{n} \in \mathbb{R}^{d}$ through some coefficient vector $\theta$, as follows:

- in vanilla linear regression it is assumed that:

- for all $i \in\{1, \ldots, n\}: y_{i}=\theta^{\top} \mathbf{x}_{i}+\epsilon_{i}$, where $\epsilon_{i} \sim \mathcal{N}(0,1)$; and

- $y_{1}, \ldots, y_{n}$ are independent.

- in vanilla logistic regression it is assumed that:

- for all $i \in\{1, \ldots, n\}$ and $\sigma_{i} \in\{ \pm 1\}: \operatorname{Pr}\left[y_{i}=\sigma_{i}\right]=$ $\frac{1}{1+\exp \left(-2 \theta^{\top} \mathbf{x}_{\mathbf{i}} \sigma_{i}\right)} ;$ and

$-y_{1}, \ldots, y_{n}$ are independent.

It is well-known that, given examples $\left(\mathbf{x}_{i}, y_{i}\right)_{i=1}^{n}$, where the $y_{i}$ 's are sampled independently as specified above, the coefficient vector $\theta$ can be estimated to within $\ell_{2}$-error $O\left(\sqrt{\frac{d}{n}}\right)$ in both models, under mild assumptions about the smallest singular value of the matrix whose rows are $\mathbf{x}_{1}, \ldots, \mathbf{x}_{n}$. In both cases, this can be achieved by solving the corresponding Maximum Likelihood Estimation (MLE) problem, which is concave. In fact, in linear regression, the optimum of the likelihood has a closed form, which is the familiar least-squares estimate.

The assumption that the response variables $y_{1}, \ldots, y_{n}$ are independent is, however, too strong. In many applications, these variables are observed on nodes of a network, or some spatial or temporal domain, and are dependent. Examples abound in financial and meteorological applications, and dependencies naturally arise in social networks through peer effects, whose study has recently exploded in topics as diverse as criminal activity (see e.g. [24]), welfare participation (see e.g. [2]), school achievement (see e.g. [36]), participation in retirement plans [18], and obesity (see e.g. [11, 39]). A prominent dataset where peer effects have been studied are data collected by the National Longitudinal Study of Adolescent Health, a.k.a. AddHealth study [26]. This was a major national study of students in grades 7-12, who were asked to name their friends-up to 10 , so that friendship networks can be constructed, and answer hundreds of questions about their personal and school life, and it also recorded information such as the age, gender, race, socio-economic background, and health of the students. Estimating models that combine peer and individual effects to predict behavior in such settings has been challenging; see e.g. [5, 31].

In this paper, we generalize the standard linear and logistic regression models to capture dependencies between the response variables, and show that if the dependencies are sufficiently weak, 
then both the coefficient vector $\theta$ and the strength of the dependencies among the response variables can be estimated to within error $O_{d}\left(\sqrt{\frac{1}{n}}\right)$. To define our models, we drop the assumption that the response variables $y_{1}, \ldots, y_{n}$ are independent, but maintain the form of the conditional distribution that each response variable $y_{i}$ takes, conditioning on a realization of the other response variables $y_{-i}$. In particular, for all $i$, conditioning on a realization of all other variables $y_{-i}$, the conditional distribution of $y_{i}$ :

- (in our linear regression model) is a Gaussian of variance 1 , as in standard linear regression, except that the mean of this Gaussian may depend on both $\theta^{\top} \mathbf{x}_{i}$ and in some restricted way the realizations $y_{j}$ and the covariates $\mathbf{x}_{j}$, for $j \neq i$;

- (in our logistic regression model) takes value +1 with probability computed by the logistic function, as in standard logistic regression, except that the logistic function is evaluated at a point that may depend on both $\theta^{\top} \mathbf{x}_{i}$ and in some restricted way the realizations $y_{j}$ and covariates $\mathbf{x}_{j}$, for $j \neq i$.

To capture network effects we parametrize the afore-described general models through a (known) interaction matrix $A \in \mathbb{R}^{n \times n}$ and an (unknown) strength of interactions $\beta \in \mathbb{R}$, as follows.

- In linear regression with $(A, \beta)$-dependent samples it is assumed that:

- $\epsilon^{\prime}=\mathbf{y}-X \theta$, with $\epsilon^{\prime} \sim \mathcal{N}\left(\mathbf{0},(\beta A+D)^{-1}\right)$.

- For all $i$, conditioning on a realization of the response variables $y_{-i}$ :

$$
y_{i}=\theta^{\top} \mathbf{x}_{i}+\epsilon_{i}^{\prime},
$$

with $\epsilon_{i}^{\prime} \sim \mathcal{N}\left(\Sigma_{i} \Sigma_{i i}^{-1} \alpha_{i}, \frac{\operatorname{det}\left((\beta A+D)_{-i}\right)}{\operatorname{det}(\beta A+D)}-\Sigma_{i} \Sigma_{i i}^{-1} \Sigma_{i}^{\top}\right)$, where $\Sigma_{i}$ is the $\mathrm{i}$-th row of $(\beta A+D)^{-1}$ by removing the coordinate (diagonal element) $i$-th, $\Sigma_{i i}$ is $(\beta A+D)^{-1}$ by removing the i-th column and $i$-th row, $(\beta A+D)_{-i}$ is $\beta A+D$ by removing $i$-th row and column and finally column vector $\alpha_{j}=y_{j}-$ $\theta^{\top} \mathbf{x}_{j}$ (this is the Schur complement for conditional multivariate Gaussians). Observe that $\Sigma_{i} \Sigma_{i i}^{-1}=-\frac{1}{D_{i i}} \beta A_{i}{ }^{1}$ and hence the expectation becomes $-\frac{1}{D_{i i}} \sum_{j \neq i} \beta A_{i j}\left(y_{j}-\theta^{\top} \mathbf{x}_{j}\right)$ and moreover the variance becomes $\frac{1}{D_{i i}}$. By the transformation $\epsilon_{i}=\epsilon_{i}^{\prime}+\frac{1}{D_{i i}} \sum_{j \neq i} \beta A_{i j}\left(y_{j}-\theta^{\top} \mathbf{x}_{j}\right)$ we get that

$$
y_{i}=\theta^{\top} \mathbf{x}_{i}-\frac{1}{D_{i i}}\left[\sum_{j \neq i} \beta A_{i j}\left(y_{j}-\theta^{\top} \mathbf{x}_{j}\right)\right]+\epsilon_{i},
$$

with $\epsilon_{i} \sim \mathcal{N}\left(0, \frac{1}{D_{i i}}\right)$.

- Interpretation: The conditional expectation of $y_{i}$ is additively perturbed from its expectation $\theta^{\top} \mathbf{x}_{i}$ by the weighted average, according to weights $\beta A_{i j}$, of how much the other responses are perturbed from their expectations in realization $y_{-j}$.

- Remark 2: The model proposed in Eq. (2) and the alternative model of Remark 1 fall in the realm of the autoregressive models studied by Manski [31] and Bramoullé et al. [5], where it is shown that the model can be identified under conditions on the interaction matrix $A$. In sharp

\footnotetext{
${ }^{1} A_{i}$ denotes the $i$-row of $A$ by removing coordinate $i$, i.e., $n-1$ vector
}

contrast to our work, one of the conditions imposed on $A$ is that it can be partitioned into many identical blocks (i.e. the weighted graph defined by $A$ has many identical connected components). Thus the response variables cluster into multiple groups that are independently and identically sampled, given the covariates. Instead we want to identify $\theta$ and $\beta$ even when $A$ corresponds to one strongly connected graph, and therefore there is no independence to be exploited.

- In logistic regression with $(A, \beta)$-dependent samples it is assumed that:

- For all $i$ and $\sigma_{i} \in\{ \pm 1\}$, conditioning on a realization of the response variables $y_{-i}$ :

$$
\begin{aligned}
\operatorname{Pr}\left[y_{i}=\sigma_{i}\right] & = \\
& \frac{1}{1+\exp \left(-2\left(\theta^{\top} \mathbf{x}_{\mathbf{i}}+\beta \sum_{j \neq i} A_{i j} y_{j}\right) \sigma_{i}\right)} .
\end{aligned}
$$

- Interpretation: The probability that the conditional distribution of $y_{i}$ assigns to +1 is determined by the logistic function applied to $2\left(\theta^{\top} \mathbf{x}_{\mathbf{i}}+\beta \sum_{j \neq i} A_{i j} y_{j}\right)$ instead of $2 \theta^{\top} \mathbf{x}_{\mathbf{i}}$, i.e. it is increased by the weighted average, according to weights $\beta A_{i j}$, of the other responses in realization $y_{-j}$.

- Remark 3: It is easy to see that the joint distribution of random variables $\left(y_{1}, \ldots, y_{n}\right)$, satisfying the requirements of Eq. (4), is an instance of the Ising model. See Eq. (6). In this Ising model each variable $i$ has external field $\theta^{\top} \mathbf{x}_{i}$, and $\beta$ controls the inverse temperature of the model. The Ising model was originally proposed to study phase transitions in spin systems [27], and has since found myriad applications in diverse research disciplines, including probability theory, Markov chain Monte Carlo, computer vision, theoretical computer science, social network analysis, game theory, and computational biology [7, 16, 19, 21, 22, 29, 35].

A particularly simple instance of our model arises when all covariates are equal and single dimensional. In this case, our model only has two free parameters, and this setting has been well-studied. [12] consider the consistency of maximum likelihood estimation in this setting. More recent work of Chatterjee [9], Bhattacharya and Mukherjee [4], and Ghosal and Mukherjee [23] has identified conditions on the interaction matrix $A$ under which these parameters can be identified. Our work generalizes these works to the case of multi-dimensional covariates.

Now let us state our results for the above regression models with dependent response variables. We are given a set of observations $\left(\mathbf{x}_{i}, y_{i}\right)_{i=1}^{n}$ where the covariates $\mathbf{x}_{i}$ are deterministic, and the response variables are assumed to have been sampled according to either of the models above, for a given interaction matrix $A$ and an unknown scalar $\beta$ and coefficient vector $\theta$. Given our observations, we are interested in estimating $\beta$ and $\theta$. It is important to stress that we only have one sample of the variables $\left(y_{1}, \ldots, y_{n}\right)$. In particular, we cannot redraw the response variables many times and derive statistical power from the independence of the samples. This is 
motivated by our application to network collected data, where we often have no access to independent snapshots of the responses at the nodes of the network. On a technical standpoint, estimating from a single sample distinguishes our work from other works in the literature of auto-regressive models and graphical models, and requires us to deal with the challenges of concentration of measure of functions of dependent random variables.

Our main result are stated as Theorems 3.1, for logistic regression, and 4.1 , for linear regression. In both cases, the parameters $\beta, \theta$ can be estimated to within error $O_{d}\left(\sqrt{\frac{1}{n}}\right)$, the dependence of the rate on $n$ matching that of vanilla regression and vanilla linear regression respectively. These results hold under the assumptions of Table 1 . We note that the assumptions on $\theta, \beta$, and the covariates are standard, even in the case of vanilla regression. Moreover, the bounds on the norm of $A$ have been shown to be necessary for logistic regression by $[4,23]$. And the minimum singular value condition for matrix $A X$ is mild, and holds for various ensembles of $A$; see e.g. Corollary 4.1 shown using Ky Fan inequalities [20].

Proof Overview: The estimation algorithms in both Theorem 3.1 and Theorem 4.1 are instances of Projected Gradient Descent (PGD). In the linear case (Theorem 4.1, PGD is applied to the negative $\log$-likelihood of the observations $\left(y_{1}, \ldots, y_{n}\right)$. However, the loglikelihood is not convex, so we perform a re-parametrization of the model, indeed an overparametrization of the model that renders it convex. Showing strong convexity of the re-parametrized negative log-likelihood requires some mild linear algebra. It has to be established that despite the overparametrization the optimum collapses to the right dimensionality, and can be used to recover the original parameters. A more complete overview of the approach is presented in the beginning of Section 4.

In the logistic case (Theorem 3.1), we do not run PGD on the negative log-likelihood but the negative

$\log$-pseudolikelihood. Pseudolikelihood is the product of the conditional probabilities of each response $y_{i}$, conditioning on all other responses $y_{-i}$. Pseudolikelihood is trivially convex, but we need to establish that is optimum is close to the true parameters and also that it is strongly convex. We show both properties via concentration results for functions of dependent random variables. To show that the maximum of the pseudolikelihood is close to the true parameters we use exchangeable pairs, adapting [10]. To show that it is strongly convex we show additional properties of $A$ which are implied by our assumptions. Combining these with a new concentration inequality, we obtain the desired bound. A more complete overview of the approach is presented in Section 3.2.

Other Related Work: We have already reviewed the work that is most relevant to ours from the Economics, Probability Theory, and Statistics literature. Further discussion of the Econometrics and Statistics literature on the theory and applications of regression with dependent observations is discussed in [30]. There is another strand of literature studying generalization bounds that can be attained when learning from sequences of dependent observations; see e.g. $[1,32-34,38,40]$. These works assume, however, that the sequence of observations is a stationary process, which does not hold in our models, and they impose strong mixing conditions on that sequence. Finally, we note that generalized linear regression, which accommodates dependencies among the response variables, cannot be applied directly to our linear regression setting to estimate $\theta$, because the covariance matrix of our response variables depends on the parameter $\beta$, which is unknown and thus needs to be disentangled before bounding the error in the estimation of $\theta$.

In the case of logistic regression, there has been a lot of work showing that under certain high-temperature conditions on the Ising model (which are similar to the assumptions we make in our paper), one can perform many statistical tasks such as learning, testing and sampling of Ising models efficiently [13-15, 17, 25, 28].

\section{PRELIMINARIES}

We use $\mathbf{x}, \mathbf{y}$ to denote random vectors and capital letters $X, Y, A, D$ to denote matrices. All vectors are assumed to be column vectors, i.e. $\operatorname{dim} \times 1$. We will overload notation slightly and refer to $A_{i j}$ as the $(i, j)^{t h}$ entry of matrix $A$. We will use the following matrix norms. For a $n \times n$ matrix $A$,

$$
\begin{gathered}
\|A\|_{2}=\max _{\|x\|_{2}=1}\|A x\|_{2}, \quad\|A\|_{\infty}=\max _{j \in[n]} \sum_{i=1}^{n}\left|A_{i j}\right|, \\
\|A\|_{F}=\sqrt{\sum_{i=1}^{n} \sum_{j=1}^{n} A_{i j}^{2} .}
\end{gathered}
$$

When $A$ is a symmetric matrix we have that $\|A\|_{2} \leq\|A\|_{\infty} \leq$ $\|A\|_{F} \leq \sqrt{n}\|A\|_{2} \leq \sqrt{n}\|A\|_{\infty}$. We use $\lambda$ to denote eigenvalues of a matrix and $\sigma$ to denote singular values. $\lambda_{\text {min }}$ refers to the smallest eigenvalue and $\lambda_{\max }$ to the largest, and similar notation for the singular values as well.

We will say an estimator $\hat{\theta}_{n}$ is consistent with a rate $\sqrt{n}$ (or equivalently $\sqrt{n}$-consistent) with respect to the true parameter $\theta_{0}$ if there exists an integer $n_{0}$ and a constant $C>0$ such that for every $n>n_{0}$, with probability at least $1-o(1)$,

$$
\left\|\hat{\theta}_{n}-\theta_{0}\right\|_{2} \leq \frac{C}{\sqrt{n}}
$$

Given an unweighted undirected graph $G(V, E)$ with adjacency matrix $A$ and assignment $\sigma: V \rightarrow\{-1,+1\}^{n}$, an Ising model is the following probability distribution on the $2^{n}$ configurations of $\sigma$ :

$$
\operatorname{Pr}\{\mathbf{y}=\sigma\}=\frac{\exp \left(\sum_{v \in V} h_{v} \sigma_{v}+\beta \sigma^{\top} A \sigma\right)}{Z_{G}(\beta, \theta)}
$$

where

$$
Z(G)=\sum_{\tilde{\sigma}} \exp \left(\sum_{v \in V} h_{v} \tilde{\sigma}_{v}+\beta \tilde{\sigma}^{\top} A \tilde{\sigma}\right)
$$

is the partition function of the system (or renormalization factor). Moreover the term $\sum_{v} h_{v} \sigma_{v}$ is called the external field. It can be observed that, without loss of generality, we can restrict the matrix $A$ to have zeros on its diagonal.

\section{LOGISTIC REGRESSION WITH DEPENDENT DATA}

In this section we look at the problem of logistic regression with dependent data. 
Table 1: List of conditions under which our main consistency results (Theorems 3.1 and 4.1) hold.

\begin{tabular}{|c|c|c|}
\hline Parameter & Logistic & Linear \\
\hline$\theta$ & {$[-\Theta, \Theta]^{d}$} & {$[-\Theta]^{d}$} \\
\hline $\begin{array}{c}\mathbf{x}_{i} \text { feature vectors with covariance } \\
\text { matrix } Q=\frac{1}{n} X^{\top} X^{2}\end{array}$ & $\begin{array}{c}\text { Support in }[-M, M]^{d} \text { and } \\
\lambda_{\max }(Q), \lambda_{\min }(Q) \text { positive } \\
\text { constants }\end{array}$ & $\begin{array}{c}\text { No restriction in the support and } \\
\lambda_{\max }(Q), \lambda_{\min }(Q) \text { positive constants }\end{array}$ \\
\hline$D$ & Not Applicable & diagonal matrix with positive constant entries \\
\hline$A$ & $\begin{array}{c}\text { symmetric, zero diagonal, } \\
\|A\|_{\infty} \leq 1 \text { and }\|A\|_{F}^{2} \geq c n\end{array}$ & $\begin{array}{c}\text { symmetric, zero diagonal, }\|A\|_{2} \leq 1 \text { and and } \\
2\end{array}$ \\
\hline$\beta$ & $(-B, B)$ & $\begin{array}{c}\lambda_{\min }\left((\beta A+D)^{-1}\right)>\rho_{\min }, \\
\lambda_{\max }\left((\beta A+D)^{-1}\right)<\rho_{\max } \text { and } \rho_{\min }, \rho_{\max } \\
\text { positive constants for all } \beta \in(-B, B)\end{array}$ \\
\hline$\frac{1}{n} X^{\top} A^{\top}\left(I-D X\left(X^{\top} D^{2} X\right)^{-1} X^{\top} D\right) A X$ & No assumption & $\begin{array}{c}\text { Minimum eigenvalue a positive constant } \\
\rho_{D A X}\end{array}$ \\
\hline
\end{tabular}

\subsection{Our Model}

We are interested in a generalization of the Ising model on graph $G=(V, E)$ with $|V|=n$, where each vertex $i \in G$ has a feature vector $\mathbf{x}_{i} \in \mathbb{R}^{d}$. Moreover there is an unknown parameter $\theta \in$ $\mathbb{R}^{d}$ and the corresponding probability distribution induces to the following:

$$
\operatorname{Pr}\{\mathbf{y}=\sigma\}=\frac{\exp \left(\sum_{i=1}^{n}\left(\theta^{\top} \mathbf{x}_{i}\right) \sigma_{i}+\beta \sigma^{\top} A \sigma\right)}{Z(G)},
$$

where $A$ is a symmetric matrix with zeros on the diagonal. Given one sample $y$ and the knowledge of the matrix $A$, we would like to infer $\beta, \theta$.

We now study some conditions under which we can attain consistent estimates of the parameters of the model. Combined with some standard assumptions on the data-generating process of the feature vectors all our assumptions are listed in Table 1.

Theorem 3.1 (Logistic Regression with Dependent SAmples). Consider the model of (6). The Maximum Pseudo-Likelihood Estimate (MPLE) $\left(\hat{\theta}_{M P L}, \hat{\beta}_{M P L}\right)$ is consistent with a rate of $\sqrt{n}$ as long as $\left(\theta_{0}, \beta_{0}\right)$ and the features $X$ satisfy the conditions of Column 2 in Table 1. Formally, for each constant $\delta>0$ and $n$ sufficiently large

$$
\left\|\left(\hat{\theta}_{M P L}, \hat{\beta}_{M P L}\right)-\left(\theta_{0}, \beta_{0}\right)\right\|_{2} \leq O_{d}\left(\sqrt{\frac{1}{n}}\right)
$$

with probability $1-\delta$. Moreover, we can compute a vector $(\tilde{\theta}, \tilde{\beta})$ with $\left\|\left(\hat{\theta}_{M P L}, \hat{\beta}_{M P L}\right)-(\tilde{\theta}, \tilde{\beta})\right\|_{2} \leq O_{d}\left(\sqrt{\frac{1}{n}}\right)$ in $O(\ln n)$ iterations of projected gradient descent (Algorithm in Section 5.1) where each iteration takes at most $O(d n)$ time, with probability $1-\delta$.

REMARK 3.1 (Necessity of AN Upper Bound on $\|A\|_{\infty}$ AND BOUNDEDNESS OF $\beta_{0}$ ). If $\|A\|_{\infty}$ scales with $n$ then no consistent estimator might exist. This is because the peer effects through $\beta_{0} A$ will dominate the outcome of the samples and will nullify the signal coming from $\theta_{0}^{\top} X$. Similarly one requires $\beta_{0}$ to be bounded as well to preserve some signal to enable recovery of $\theta_{0}$.

Remark 3.2 (Necessity of THE Lower Bound on $\|A\|_{F}$ ). It was shown in [4] (Corollary 2.4 (b)) and [23] (Theorem 1.13) that when the condition $\|A\|_{F}^{2}>$ cn is violated, we have specific examples where it is impossible to get consistent estimators for $\left(\theta_{0}, \beta_{0}\right)$. The first instance is the Curie-Weiss model $C W(n, \beta, h)\left(A_{i j}=\frac{1}{n}\right.$ for all $\left.i \neq j\right)$. Note that $\|A\|_{F}^{2}=O(1)$ in this case. The second instance is dense random graphs, i.e. $G(n, p)$ where $p$ is a constant independent of $n$ and $A$ is chosen to be the adjacency matrix scaled down by the average degree of the graph, i.e. $A_{i j}=\frac{1}{(n-1) p} \mathbb{1}_{(i, j) \in E}$.

REMARK 3.3. If the parameter $\beta_{0}$ is known, the condition that $\|A\|_{F}^{2} \geq \mathrm{cn}$ is not necessary for consistency of the MPL estimate $\hat{\theta}_{M P L}$. For instance, consider the independent case where $\beta_{0}=0$. Then, to recover $\theta$, we do not need $\|A\|_{F}^{2} \geq c n$.

REMARK 3.4. Our approach achieves a $\sqrt{n / d}$ rate of consistency if $\|\mathbf{x}\|_{2} \times\|\theta\|_{2}=O(1)$. This is the rate one gets in the regime with independent samples.

Example Instantiations of Theorem 3.1 Two example settings where the conditions required for Theorem 3.1 to hold are satisfied are

- $A$ is the adjacency matrix of graphs with bounded degree $d$ scaled down so that $\|A\|_{2} \leq 1$.

- $A$ is the adjacency matrix of a random $d$-regular graph.

\subsection{Technical Overview}

Estimation in Ising models is a well-studied problem which offers a lot of interesting technical challenges. A first approach one considers is maximum likelihood estimation. However the intractability of computing the partition function poses a serious obstacle for the MLE. Even if one could approximate the partition function, proving consistency of the MLE is a hard task. To circumvent these issues we take a maximum pseudo-likelihood approach. This was proposed by Julian Besag [3] and analyzed for inference problems on Ising models by Chatterjee [9] and others ([4],[23]). Given a sample of response variables y let $f_{i}(\theta, \beta, y)$ denote the condition likelihood of observing $y_{i}$ conditioned on everyone else. The pseudo-likelihood estimator of $\mathbf{y}$ is

$$
\left(\hat{\theta}_{M P L}, \hat{\beta}_{M P L}\right)=\operatorname{argmax}_{\theta, \beta} \prod_{i=1}^{n} f_{i}(\theta, \beta, \mathbf{y}) .
$$


This does away with the problematic partition function and retains concavity in the parameters $\theta, \beta$. To show that the MPLE is consistent we need to show that its global optimum $\left(\hat{\theta}_{M P L}, \hat{\beta}_{M P L}\right)$ is close in $\ell_{2}$ distance to $\left(\theta_{0}, \beta_{0}\right)$. We achieve this by showing two things hold simultaneously.

- The log pseudo-likelihood is strongly concave everywhere. This will tell us that the gradient of the log pseudo-likelihood quickly increases as we move away from $\left(\hat{\theta}_{M P L}, \hat{\beta}_{M P L}\right)$ where it is 0 .

- The norm of the gradient of the log pseudo-likelihood is small at when evaluated at $\left(\theta_{0}, \beta_{0}\right)$ hence implying proximity to the MPL estimates due to strong concavity.

We show that both these conditions are satisfied with high probability over the draw of our samples. Showing that the norm of the gradient is bounded involves obtaining variance bounds on two functions of the Ising model (Lemmas 3.2 and 3.3), and showing strong concavity amounts to showing a linear in $n$ lower bound on a particular quadratic function (Lemma 3.4). Both these properties are challenging to prove because of the dependences between samples. To tackle the lack of independence, the proofs require a rich set of technical frameworks. In particular, to show the variance bounds we use the technique of exchangeable pairs developed by Chatterjee [8]. The boundedness of $\|A\|_{\infty}$ is necessary to have these concentration results. To show strong concavity of the log pseudolikelihood we first prove some properties of the matrix $A$ together with an additional variance bound again shown via exchangeable pairs. The lower bound on $\|A\|_{F}$ is necessary to achieve strong concavity. Finally, we show in Section 5 that computing the MPLE can be achieved efficiently using projected gradient descent where after each step we project back into the space restriced by the conditions of Table 1 . We describe each of these steps formally now.

\subsection{Analyzing the Maximum Pseudolikelihood Estimator (MPLE)}

We will treat terms not involving $n$ as constants for the purposes of our analysis. We start by analyzing the maximum pseudo-likelihood estimator. Given the feature vector of the $i^{t h}$ sample $\mathbf{x}_{i}$, we denote by $x_{i k}$ the $k^{t h}$ element of $\mathbf{x}_{i}$. The $\log$ pseudolikelihood for a specific sample $\mathbf{y}$ is given by:

$$
\begin{aligned}
& L P L(\theta, \beta):=-\ln 2+ \\
& \frac{1}{n} \sum_{i=1}^{n}\left[y_{i} \beta m_{i}(\mathbf{y})+y_{i}\left(\theta^{\top} \mathbf{x}_{i}\right)-\ln \cosh \left(\beta m_{i}(\mathbf{y})+\theta^{\top} \mathbf{x}_{i}\right)\right],
\end{aligned}
$$

where $m_{i}(\mathbf{y}):=\sum_{j=1}^{n} A_{i j} y_{j}$.

The first order conditions give:

$$
\begin{aligned}
& \frac{\partial L P L(\theta, \beta)}{\partial \beta}= \\
& =\frac{1}{n} \sum_{i=1}^{n}\left[y_{i} m_{i}(\mathbf{y})-m_{i}(\mathbf{y}) \tanh \left(\beta m_{i}(\mathbf{y})+\theta^{\top} \mathbf{x}_{i}\right)\right]=0 \\
& \frac{\partial L P L(\theta, \beta)}{\partial \theta_{k}}= \\
& =\frac{1}{n} \sum_{i=1}^{n}\left[y_{i} x_{i, k}-x_{i, k} \tanh \left(\beta m_{i}(\mathbf{y})+\theta^{\top} \mathbf{x}_{i}\right)\right]=0
\end{aligned}
$$

Say the solution to equation (9) is $(\hat{\theta}, \hat{\beta})$.
The Hessian $H_{(\theta, \beta)}$ is given by:

$$
\begin{aligned}
& \frac{\partial^{2} L P L(\theta, \beta)}{\partial \beta^{2}}=-\frac{1}{n} \sum_{i=1}^{n} \frac{m_{i}^{2}(\mathrm{y})}{\cosh ^{2}\left(\beta m_{i}(\mathbf{y})+\theta^{\top} \mathbf{x}_{i}\right)}, \\
& \frac{\partial^{2} L P L(\theta, \beta)}{\partial \beta \partial \theta_{k}}=-\frac{1}{n} \sum_{i=1}^{n} \frac{x_{i, k} m_{i}(\mathbf{y})}{\cosh ^{2}\left(\beta m_{i}(\mathbf{y})+\theta^{\top} \mathbf{x}_{i}\right)}, \\
& \frac{\partial^{2} L P L(\theta, \beta)}{\partial \theta_{l} \partial \theta_{k}}=-\frac{1}{n} \sum_{i=1}^{n} \frac{x_{i, l} x_{i, k}}{\cosh ^{2}\left(\beta m_{i}(\mathbf{y})+\theta^{\top} \mathbf{x}_{i}\right)} .
\end{aligned}
$$

Writing the Hessian in a compact way we get

$$
H_{(\theta, \beta)}=-\frac{1}{n} \sum_{i=1}^{n} \frac{1}{\cosh ^{2}\left(\beta m_{i}(\mathbf{y})+\theta^{\top} \mathbf{x}_{i}\right)} X_{i} X_{i}^{\top}
$$

where $X_{i}=\left(\mathbf{x}_{i}, m_{i}(\mathbf{y})\right)^{\top}$. Thus $-H$ is a positive semidefinite matrix and LPL is concave. Moreover if $(\theta, \beta) \in \mathbb{B}$ it follows that

$$
\begin{aligned}
& \frac{1}{\cosh ^{2}(B+d \cdot M \cdot \Theta)} \cdot\left(\frac{1}{n} \sum_{i=1}^{n} X_{i} X_{i}^{\top}\right) \leq-H_{(\theta, \beta)} \\
& \leq\left(\frac{1}{n} \sum_{i=1}^{n} X_{i} X_{i}^{\top}\right) .
\end{aligned}
$$

REMARK 3.5. Observe that $\left\|X_{i}\right\|_{2}^{2}=\left\|x_{i}\right\|_{2}^{2}+m_{i}^{2}(\mathrm{y}) \leq d \Theta^{2}+1$ (assuming that $\|A\|_{\infty} \leq 1$ trivially holds $\left|m_{i}(x)\right| \leq 1$ ). It is easy to see that $\lambda_{\max }\left(-H_{(\theta, \beta)}\right) \leq \sqrt{d \Theta^{2}+1}$ for all $(\theta, \beta) \in \mathbb{R}^{d+1}$, hence $-L P L$ is $a \sqrt{d \Theta^{2}+1}$-smooth function, i.e. $\nabla-L P L$ is $\sqrt{d \Theta^{2}+1}$-Lipschitz.

\subsection{Consistency of the MPLE}

Our argument for showing consistency of the MPLE uses Lemma 3.1.

Lemma 3.1. Let $\left(\theta_{0}, \beta_{0}\right)$ be the true parameter and assume $\mathbb{B}=$ $[-\Theta, \Theta]^{d} \times[-B, B]$ (the true parameter is in the interior of $\left.\mathbb{B}\right)$. We define $\left(\theta_{t}, \beta_{t}\right)=(1-t)\left(\theta_{0}, \beta_{0}\right)+t\left(\hat{\theta}_{M P L}, \hat{\beta}_{M P L}\right)$ and let $\mathcal{D} \in[0,1]$ be the largest value such that $\left(\theta_{\mathcal{D}}, \beta_{\mathcal{D}}\right) \in \mathbb{B}$ (if it does not intersect $\mathbb{B}$, then $\mathcal{D}=1$ ). Then,

$$
\begin{aligned}
& \left\|\nabla L P L\left(\theta_{0}, \beta_{0}\right)\right\|_{2} \\
& \geq \mathcal{D} \min _{(\theta, \beta) \in \mathbb{B}} \lambda_{\min }\left(-H_{(\theta, \beta)}\right)\left\|\left(\theta_{0}-\hat{\theta}_{M P L}, \beta_{0}-\hat{\beta}_{M P L}\right)\right\|_{2} \\
& =\min _{(\theta, \beta) \in \mathbb{B}} \lambda_{\min }\left(-H_{(\theta, \beta)}\right)\left\|\left(\theta_{0}-\theta_{\mathcal{D}}, \beta_{0}-\beta_{\mathcal{D}}\right)\right\|_{2}
\end{aligned}
$$

Proof. We drop the subscript $M P L$ from the estimates for brevity. We set

$$
\begin{gathered}
g(t):=\left(\theta_{0}-\hat{\theta}, \beta_{0}-\hat{\beta}\right)^{\top} \nabla L P L\left(\theta_{t}, \beta_{t}\right), \\
g^{\prime}(t)=-\left(\theta_{0}-\hat{\theta}, \beta_{0}-\hat{\beta}\right)^{\top} H_{\left(\theta_{t}, \beta_{t}\right)}\left(\theta_{0}-\hat{\theta}, \beta_{0}-\hat{\beta}\right) .
\end{gathered}
$$

Observe that $\mathcal{D}=\frac{\left\|\left(\theta_{\mathfrak{D}}-\theta_{0}, \beta_{\mathfrak{D}}-\beta_{0}\right)\right\|_{2}}{\left\|\left(\hat{\theta}-\theta_{0}, \hat{\beta}-\beta_{0}\right)\right\|_{2}}$. Since $H$ is negative semidefinite we have that $g^{\prime}(t) \geq 0\left(^{*}\right)$. It holds that

$$
\begin{aligned}
& \left\|\left(\theta_{0}-\hat{\theta}, \beta_{0}-\hat{\beta}\right)\right\|_{2} \cdot\left\|\nabla L P L\left(\theta_{0}, \beta_{0}\right)\right\|_{2} \\
& \geq\left|\left(\theta_{0}-\hat{\theta}, \beta_{0}-\hat{\beta}\right)^{\top} \nabla L P L\left(\theta_{0}, \beta_{0}\right)\right| \\
& =|g(1)-g(0)| \\
& =\left|\int_{0}^{1} g^{\prime}(t) d t\right| \\
& \geq\left|\int_{0}^{\mathcal{D}} g^{\prime}(t) d t\right| \text { by }\left(^{*}\right) \\
& \geq \mathcal{D} \min _{(\theta, \beta) \in \mathbb{B}} \lambda_{\min }\left(-H_{(\theta, \beta)}\right)\left\|\left(\theta_{0}-\hat{\theta}, \beta_{0}-\hat{\beta}\right)\right\|_{2}^{2}
\end{aligned}
$$




$$
\begin{aligned}
& =\min _{(\theta, \beta) \in \mathbb{B}} \lambda_{\min }\left(-H_{(\theta, \beta)}\right)\left\|\left(\theta_{\mathcal{D}}-\theta_{0}, \beta_{\mathcal{D}}-\beta_{0}\right)\right\|_{2} \times \\
& \times\left\|\left(\theta_{0}-\hat{\theta}, \beta_{0}-\hat{\beta}\right)\right\|_{2}
\end{aligned}
$$

We apply Lemma 3.1 by showing a concentration result for $\left\|\nabla L P L\left(\theta_{0}, \beta_{0}\right)\right\|_{2}^{2}$ around $1 / n$ and a (positive constant) lower bound for $\min _{(\theta, \beta) \in \mathbb{B}} \lambda_{\min }\left(-H_{(\theta, \beta)}\right)$ combining with the observation that $D \rightarrow 1^{3}$ as $n \rightarrow \infty$ (i.e., $D \geq \frac{1}{2}$ for $n$ sufficiently large) to give the desired rate of consistency.

\subsection{Variance Bounds using Exchangeable Pairs}

In this Section we state the lemma which are required to show that the norm of the gradient of the log pseudo-likelihood is bounded at the true parameters.

Lemma 3.2 (Variance Bound 1). It holds that

$$
\begin{aligned}
& \mathbb{E}_{\theta_{0}, \beta_{0}}\left[\left(\sum_{i=1}^{n} y_{i} m_{i}(\mathbf{y})-m_{i}(\mathbf{y}) \tanh \left(\beta_{0} m_{i}(\mathbf{y})+\theta_{0}^{\top} \mathbf{x}_{i}\right)\right)^{2}\right] \\
& \leq(12+4 B) n .
\end{aligned}
$$

Lemma 3.3 (VARIANCE Bound 2).

$$
\begin{aligned}
& \mathbb{E}_{\theta_{0}, \beta_{0}}\left[\sum_{k=1}^{d}\left(\sum_{i=1}^{n} x_{i, k} y_{i}-x_{i, k} \tanh \left(\beta_{0} m_{i}(\mathbf{y})+\theta_{0}^{\top} \mathbf{x}_{i}\right)\right)^{2}\right] \\
& \leq(4+4 B) M^{2} \cdot d n .
\end{aligned}
$$

The proofs of the above Lemmas adapts the technique of exchangeable pairs and are deferred to full version of this paper.

\subsection{Strong Concavity of Maximum Pseudolikelihood}

We show the following result which will imply that the negative log pseudo-likelihood is strongly convex everywhere with a large probability.

Lemma 3.4. Let $F=I-X\left(X^{\top} X\right)^{-1} X^{\top}$. Suppose $\|A\|_{F}^{2} \geq C n$, then there exist constants $c$ and $\delta$ such that,

$$
\operatorname{Pr}_{\theta_{0}, \beta_{0}}\left[\sum_{i}(F A y)_{i}^{2} \geq c n\right] \geq 1-\delta .
$$

\section{LINEAR REGRESSION WITH DEPENDENT OBSERVATIONS}

In this section we focus on linear regression under weakly dependent errors. As opposed to Logistic regression, in linear regression the log-likelihood is computationally tractable. This section is devoted to showing that the Maximum Likelihood Estimator (MLE) under appropriate (over)reparametrization - the new parameter vector will be $(\theta, \beta, \kappa)$ where $\theta, \beta$ remain same after reparametrization and $\kappa=\beta . \theta$ - is $\sqrt{n}$ consistent in our linear regression model. We set $\mathbb{B}=[-\Theta, \Theta]^{d} \times[-B, B] \times[-\Theta B, \Theta B]^{d}$. (the set that the parameters

\footnotetext{
${ }^{3}$ This is true because $\left\|\left(\theta_{\mathcal{D}}-\theta_{0}, \beta_{\mathcal{D}}-\beta_{0}\right)\right\|_{2} \rightarrow 0$ as $n \rightarrow \infty$ (is of order $\frac{1}{\sqrt{n}}$ by showing the promised concentration result and the lower bound).
}

should be in and $B$ is defined in Table 1). Formally we prove the following theorem.

Theorem 4.1 (MAIn Linear). Given a

(1) feature matrix $X$ with $Q=\frac{1}{n} X^{\top} X$ having $\lambda_{\min }(Q), \lambda_{\max }(Q)$ as positive constants ${ }^{4}$, and

(2) $\|A\|_{2}=\Theta(1),\|A\|_{F}^{2}=\Omega(n)$ and

$$
\lambda_{\min }\left(\frac{1}{n} X^{\top} A^{\top}\left(I-D X\left(X^{\top} D^{2} X\right)^{-1} X^{\top} D\right) A X\right)=\Theta(1) .
$$

The Maximum Log-likelihood Estimate (MLE) $(\hat{\theta}, \hat{\beta}, \hat{\kappa})$ is $O_{d}\left(\sqrt{\frac{1}{n}}\right)$ consistent as long as the true parameter vector $\left(\theta_{0}, \beta_{0}, \kappa_{0}\right) \in \mathbb{B}$ (interior of $\left.\mathbb{B}\right)$, i.e., for each $\delta>0$ and $n$ sufficiently large, $\left\|(\hat{\theta}, \hat{\beta}, \hat{\kappa})-\left(\theta_{0}, \beta_{0}, \kappa_{0}\right)\right\|_{2}$ is $O_{d}\left(\sqrt{\frac{1}{n}}\right)$ with probability $1-\delta$. Moreover, we can compute a vector $(\tilde{\theta}, \tilde{\beta})$ with $\|(\hat{\theta}, \hat{\beta})-(\tilde{\theta}, \tilde{\beta})\|_{2}$ to be $O_{d}\left(\sqrt{\frac{1}{n}}\right)$ in $O(\ln n)$ iterations of projected gradient descent $t^{5}$ with probability $1-o(1)$.

Corollary 4.1 (Sherrington-Kirkpatrick (S-K) MODEL). In the Sherrington-Kirkpatrick model [37], we have that $A_{i j}=\frac{g_{i j}}{\sqrt{n}}$ for $i<j$, where $g_{i j} \sim \mathcal{N}(0,1)$ and $A_{j i}=A_{i j}, A_{i i}=0$. It can be shown that $A$ satisfies the assumptions of our main theorem, so we can infer $\beta, \theta$ (with a $\sqrt{n}$ rate of consistency).

Technically, we will reparametrize the log-likelihood function in such a way that the new parameter vector is not high-dimensional and the resulting log-likelihood becomes strongly convex. The reparametrization and the equations of log-likelihood, its gradient and Hessian can be found in Section 4.1. We will follow the same high level ideas as in the logistic regression. Under assumptions on $A, \beta, \theta,(\beta A+D)^{-1}, A X$ that are summarized in Table 1 we proceed as follows:

- We prove concentration results for the gradient of the re parametrized log-likelihood, see Lemmas 4.3,4.1 and 4.2 in Section 4.2.1.

- We prove that the minimum eigenvalue of the negative Hessian of reparametrized log-likelihood is large enough, see Lemma 4.4 in Section 4.2.2.

REMARK 4.1. Similar to Remark 3.4, our approach for linear regression achieves $a \sqrt{n / d}$ rate of consistency if $\|\mathbf{x}\|_{2} \times\|\theta\|_{2}=O(1)$. This is the rate one gets in the regime with independent samples.

Below we provide some important definitions.

Gaussian Graphical Model. Let $G=(V, E)$ be an undirected graph with $V=[n]$. A random vector $X \in \mathbb{R}^{n}$ is said to be distributed according to (undirected) Gaussian Graphical model with graph $G$ if $X$ has a multivariate Gaussian distribution $\mathcal{N}(\mu, \Sigma)$ with

$$
\left(\Sigma^{-1}\right)[i, j]=0 \quad \forall(i, j) \notin E,
$$

where the density function $f_{\mu, \Sigma}($.$) of \mathcal{N}(\mu, \Sigma)$ is

$$
f_{\mu, \Sigma}(x)=\frac{\exp \left(-\frac{1}{2}(x-\mu)^{T} \Sigma^{-1}(x-\mu)\right)}{(2 \pi)^{n / 2} \operatorname{det}(\Sigma)^{1 / 2}}
$$

\footnotetext{
$\mathbf{x}_{i}$ can be subgaussian.

${ }^{5}$ Each iteration is polynomial time computable
} 
under the condition that $\Sigma$ is positive semi-definite $\left(\Sigma^{-1}\right.$ is also known as the precision matrix).

In our setting, we have that the errors $\epsilon_{i}=y_{i}-\theta x_{i}$ are distributed according to a Gaussian graphical model. Since each $\epsilon_{i}$ is zero mean, we have that $\mu=0$ in our case. Also, similar to the logistic regression setting, we will assume that we have complete knowledge of the graph structure up to a scaling factor. That is, $(\Sigma)^{-1}=\beta A+D$ where the matrix $A$ is a known symmetric matrix with $A_{i i}=0$ and $D=\left[d_{1} \ldots d_{n}\right]^{T}$ is a known diagonal matrix with positive entries.

\subsection{Our Reparametrization and Log-likelihood}

It is not hard to see that the negative log-likelihood is not convex with respect to the parameter vector $(\theta, \beta) \in \mathbb{R}^{d+1}$, for the linear regression model with dependent errors. Nevertheless, we can reparametrize the log-likelihood in such a way to make it convex. The classic way to do it sets $T:=\Sigma^{-1}, v=\Sigma^{-1} \mu$ (for a gaussian $\mathcal{N}(\mu, \Sigma)$ ). However, this creates a parameter vector of dimension $\Omega(n)$. It is crucial that after the reparametrization the dimensionality of the new parameter vector is $O(d)$ and not $\Omega(n)$ (for concentration purposes, see remark 4.2). Hence, we take a different route here.

We set $\kappa:=\beta \cdot \theta \in \mathbb{R}^{d}$ and define our parametric vector to be $(\theta, \beta, \kappa) \in \mathbb{R}^{2 d+1}$ (parameters $\theta, \beta$ remain the same and we introduce vector $\kappa)$. The vector $(\theta, \beta, \kappa)$ is $(2 d+1)$-dimensional. Our reparameterization is an overparameterization which helps us achieve convexity of the negative log-likelihood function.

The negative log-likelihood is given by the following:

$$
\begin{aligned}
-L L & =\frac{1}{2}(\mathbf{y}-X \theta)^{\top}(\beta A+D)(\mathbf{y}-X \theta)+ \\
& \log \int_{\mathbb{R}^{n}} \exp \left(-\frac{1}{2}(\mathbf{z}-X \theta)^{\top}(\beta A+D)(\mathbf{z}-X \theta)\right) d z \\
& =\frac{1}{2} \mathbf{y}^{\top}(\beta A+D) \mathbf{y}-\mathbf{y}^{\top} A X \kappa-\mathbf{y}^{\top} D X \theta+ \\
& \log \int_{\mathbb{R}^{n}} \exp \left(-\frac{1}{2} \mathbf{z}^{\top}(\beta A+D) \mathbf{z}+\mathbf{z}^{\top} A X \kappa+\mathbf{z}^{\top} D X \theta\right) d z
\end{aligned}
$$

The negative gradient of the log-likelihood is given below:

$$
\begin{aligned}
& -\nabla_{\theta} L L(\theta, \beta, \kappa)=-\mathbf{y}^{\top} D X+ \\
& \frac{\int_{\mathbb{R}^{n}} \mathbf{z}^{\top} D X \exp \left(-\frac{1}{2} \mathbf{z}^{\top}(\beta A+D) \mathbf{z}+\mathbf{z}^{\top} A X \kappa+\mathbf{z}^{\top} D X \theta\right) d z}{\int_{\mathbb{R}^{n}} \exp \left(-\frac{1}{2} \mathbf{z}^{\top}(\beta A+D) \mathbf{z}+\mathbf{z}^{\top} A X \kappa+\mathbf{z}^{\top} D X \theta\right) d z} \\
& -\nabla_{\beta} L L(\theta, \beta, \kappa)=\frac{1}{2} \mathbf{y}^{\top} A \mathbf{y}+ \\
& \frac{\int_{\mathbb{R}^{n}}-\frac{1}{2} \mathbf{z}^{\top} A \mathbf{z} \exp \left(-\frac{1}{2} \mathbf{z}^{\top}(\beta A+D) \mathbf{z}+\mathbf{z}^{\top} A X \kappa+\mathbf{z}^{\top} D X \theta\right) d z}{\int_{\mathbb{R}^{n}} \exp \left(-\frac{1}{2} \mathbf{z}^{\top}(\beta A+D) \mathbf{z}+\mathbf{z}^{\top} A X \kappa+\mathbf{z}^{\top} D X \theta\right) d z} \\
& -\nabla_{\kappa} L L(\theta, \beta, \kappa)=-\mathbf{y}^{\top} A X+ \\
& \frac{\int_{\mathbb{R}^{n}} \mathbf{z}^{\top} A X \exp \left(-\frac{1}{2} \mathbf{z}^{\top}(\beta A+D) \mathbf{z}+\mathbf{z}^{\top} A X \kappa+\mathbf{z}^{\top} D X \theta\right) d z}{\int_{\mathbb{R}^{n}} \exp \left(-\frac{1}{2} \mathbf{z}^{\top}(\beta A+D) \mathbf{z}+\mathbf{z}^{\top} A X \kappa+\mathbf{z}^{\top} D X \theta\right) d z} .
\end{aligned}
$$

The negative hessian of the log-likelihood is given below (it is of size $(2 d+1) \times(2 d+1))$ :

$$
\begin{aligned}
& -H:=-\nabla^{2} L L=\operatorname{Cov}_{z \sim \mathcal{N}\left((\beta A+D)^{-1}(A X \kappa+D X \theta),(\beta A+D)^{-1}\right)} \\
& {\left[\left(\begin{array}{c}
-\frac{1}{2} \mathbf{z}^{\top} A \mathbf{z} \\
X^{\top} D \mathbf{z} \\
X^{\top} A \mathbf{z}
\end{array}\right),\left(\begin{array}{c}
-\frac{1}{2} \mathbf{z}^{\top} A \mathbf{z} \\
X^{\top} D \mathbf{z} \\
X^{\top} A \mathbf{z}
\end{array}\right)\right] .}
\end{aligned}
$$

\subsection{Consistency of Likelihood}

Let $\left(\theta_{0}, \beta_{0}, \kappa_{0}\right)$ be the true parameter (observe that $\left.\kappa_{0}=\beta_{0} \cdot \theta_{0}\right)$ and assume $\mathbb{B}=[-\Theta, \Theta]^{d} \times[-B, B] \times[-\Theta B, \Theta B]^{d}$ (true parameter lies in the interior of $\mathbb{B})$. We define $\left(\theta_{t}, \beta_{t}, \kappa_{t}\right)=(1-t)\left(\theta_{0}, \beta_{0}, \kappa_{0}\right)+$ $t(\hat{\theta}, \hat{\beta}, \hat{\kappa})$ where $(\hat{\theta}, \hat{\beta}, \hat{\kappa})$ satisfies the first order conditions for $L L$ (i.e., $\nabla L L(\hat{\theta}, \hat{\beta}, \hat{\kappa})=0)^{6}$ and set

$$
\begin{gathered}
g(t):=\left(\theta_{0}-\hat{\theta}, \beta_{0}-\hat{\beta}, \kappa_{0}-\hat{\kappa}\right)^{\top} \nabla L L\left(\theta_{t}, \beta_{t}, \kappa_{t}\right), \\
g^{\prime}(t)=-\left(\theta_{0}-\hat{\theta}, \beta_{0}-\hat{\beta}, \kappa_{0}-\hat{\kappa}\right)^{\top} H_{\left(\theta_{t}, \beta_{t}, \kappa_{t}\right)}\left(\theta_{0}-\hat{\theta}, \beta_{0}-\hat{\beta}, \kappa_{0}-\hat{\kappa}\right) .
\end{gathered}
$$

Let $\mathcal{D} \in[0,1]$ be such that $\left(\theta_{\mathcal{D}}, \beta_{\mathcal{D}}, \kappa_{\mathcal{D}}\right)$ intersects the $\tilde{\mathbb{B}}$ set (if it does not intersect $\mathbb{B}$ then $\mathcal{D}=1)$. Since $H$ is negative semidefinite (from analysis in equation 23) we have that $g^{\prime}(t) \geq 0\left(^{* *}\right)$. It holds that

$$
\begin{aligned}
& \left\|\left(\theta_{0}-\hat{\theta}, \beta_{0}-\hat{\beta}, \kappa_{0}-\hat{\kappa}\right)\right\|_{2} \cdot\left\|\nabla L L\left(\theta_{0}, \beta_{0}, \kappa_{0}\right)\right\|_{2} \\
& \geq\left|\left(\theta_{0}-\hat{\theta}, \beta_{0}-\hat{\beta}, \kappa_{0}-\hat{\kappa}\right)^{\top} \nabla L L\left(\theta_{0}, \beta_{0}, \kappa_{0}\right)\right| \\
& =|g(1)-g(0)| \\
& =\left|\int_{0}^{1} g^{\prime}(t) d t\right| \\
& \geq\left|\int_{0}^{\mathcal{D}} g^{\prime}(t) d t\right| \operatorname{by}\left(^{* *}\right) \\
& \geq \mathcal{D} \min _{(\theta, \beta, \kappa) \in \mathbb{B}} \lambda_{\min }\left(-H_{(\theta, \beta, \kappa)}\right)\left\|\left(\theta_{0}-\hat{\theta}, \beta_{0}-\hat{\beta}, \kappa_{0}-\hat{\kappa}\right)\right\|_{2}^{2} .
\end{aligned}
$$

The aforementioned inequalities indicate that we need a concentration result for $\left\|\frac{1}{n} \nabla L L\left(\theta_{0}, \beta_{0}, \kappa_{0}\right)\right\|_{2}$ and a lower bound on the minimum eigenvalue of $-\frac{1}{n} H$ for consistency of the MLE. As in the logistic regression case, combining with the observation that $D \rightarrow 1^{7}$ as $n \rightarrow \infty$ (i.e., $D \geq \frac{1}{2}$ for $n$ sufficiently large) we get the desired rate of consistency.

\subsubsection{Concentration results. We have}

$$
\begin{aligned}
& \mathbb{E}_{\theta_{0}, \beta_{0}}\left[\left\|\nabla L L\left(\theta_{0}, \beta_{0}, \beta_{0} \cdot \theta_{0}\right)\right\|_{2}^{2}\right]= \\
& \mathbb{E}_{\theta_{0}, \beta_{0}}\left[\left\|\nabla_{\theta} L L\left(\theta_{0}, \beta_{0}, \beta_{0} \cdot \theta_{0}\right)\right\|_{2}^{2}\right]+ \\
& \mathbb{E}_{\theta_{0}, \beta_{0}}\left[\left|\nabla \nabla_{\beta} L L\left(\theta_{0}, \beta_{0}, \beta_{0} \cdot \theta_{0}\right)\right|^{2}\right]+ \\
& \mathbb{E}_{\theta_{0}, \beta_{0}}\left[\left\|\nabla_{\kappa} L L\left(\theta_{0}, \beta_{0}, \beta_{0} \cdot \theta_{0}\right)\right\|_{2}^{2}\right] .
\end{aligned}
$$

We prove below concentration results for each term separately.

LEMMA 4.1 (BOUNDING THE 1ST TERM).

$$
\begin{gathered}
\mathbb{E}_{\theta_{0}, \beta_{0}}\left[\left\|\nabla_{\theta} L L\left(\theta_{0}, \beta_{0}, \kappa_{0}\right)\right\|_{2}^{2}\right]=\left\|\left(\beta_{0} A+D\right)^{-1 / 2} D X\right\|_{F}^{2} \leq \\
\left\|\left(\beta_{0} A+D\right)^{-1 / 2} D\right\|_{2}^{2}\|X\|_{F}^{2} .
\end{gathered}
$$

LEMma 4.2 (Bounding THE 3RD TERM). Similarly to Lemma 4.1 we get

$$
\begin{gathered}
\mathbb{E}_{\theta_{0}, \beta_{0}}\left[\left\|\nabla_{\kappa} L L\left(\theta_{0}, \beta_{0}, \kappa_{0}\right)\right\|_{2}^{2}\right]=\left\|\left(\beta_{0} A+D\right)^{-1 / 2} A X\right\|_{F}^{2} \\
\leq\left\|\left(\beta_{0} A+D\right)^{-1 / 2} A\right\|_{2}^{2}\|X\|_{F}^{2} .
\end{gathered}
$$

\footnotetext{
${ }^{6}$ Observe that is not necessarily true that $\hat{\kappa}=\hat{\beta} \hat{\theta}$.

${ }^{7}$ This is true because $\left\|\left(\theta_{\mathcal{D}}-\theta_{0}, \beta_{\mathcal{D}}-\beta_{0}, \kappa_{\mathcal{D}}-\kappa_{0}\right)\right\|_{2} \rightarrow 0$ as $n \rightarrow \infty$ by showing the promised concentration result and the lower bound).
} 
LEMMA 4.3 (BOUNDING THE 2ND TERM). It holds that

$$
\begin{gathered}
\mathbb{E}_{\theta_{0}, \beta_{0}, \kappa_{0}}\left[\left|\nabla_{\beta} L L\left(\theta_{0}, \beta_{0}, \kappa_{0}\right)\right|^{2}\right] \leq \\
2\left\|\left(\beta_{0} A+D\right)^{-1 / 2} A\left(\beta_{0} A+D\right)^{-1 / 2}\right\|_{F}^{2}+ \\
4 d \Theta^{2}\left\|\left(\beta_{0} A+D\right)^{-1 / 2} A X\right\|_{2}^{2} .
\end{gathered}
$$

REMARK 4.2. We note the dependence on the dimension $d$ for the bound in Lemma 4.3. This indicates how crucial it is that the dimensionality of the parameter vector does not scale with $n$.

4.2.2 Lower bound on the minimum eigenvalue. In this section we provide a lower bound on the minimum eigenvalue of the negative Hessian of the log-likelihood. We need this bound for strong concavity of the log-likelihood.

Lemma 4.4 (Bounding the Minimum eigenvalue). Let $z \sim$ $\mathcal{N}\left((\beta A+D)^{-1}(A X \kappa+D X \theta),(\beta A+D)^{-1}\right)$. There exists a constant $C$ such that

$$
\lambda_{\min }\left(\operatorname{Cov}\left[\left(\begin{array}{c}
-\frac{1}{2} \mathbf{z}^{\top} A \mathbf{z} \\
X^{\top} D \mathbf{z} \\
X^{\top} A \mathbf{z}
\end{array}\right),\left(\begin{array}{c}
-\frac{1}{2} \mathbf{z}^{\top} A \mathbf{z} \\
X^{\top} D \mathbf{z} \\
X^{\top} A z
\end{array}\right)\right]\right) \geq C n .
$$

Remark 4.3 (Smoothness of Hessian). An upper bound on the eigenvalues of the negative Hessian would be that

$$
\lambda_{\max }\left(-H_{\theta, \beta, \kappa}\right) \leq C_{H} n
$$

for all $(\theta, \beta, \kappa) \in \mathbb{B}$ for some positive constant $C_{H}$.

We conclude by proving the main theorem:

Proof of Theorem 4.1. For any $\delta>0$ and using Markov's inequality it follows from Lemmas 4.1, 4.3, 4.2 and facts about the eigenvalues of $\beta A+D, X^{\top} X$ that

$$
\underset{\theta_{0}, \beta_{0}}{\operatorname{Pr}}\left[\left\|\nabla L L\left(\theta_{0}, \beta_{0}, \beta_{0} \cdot \theta_{0}\right)\right\|_{2} \geq C_{\delta} \sqrt{d n}\right] \leq \delta
$$

for some constant $C_{\delta}$ and $\lambda_{\min }\left(\nabla^{2} L L\right) \geq C$ for some constant $C$. We conclude from the analysis in Section 4.2 that $\left\|\left(\theta_{0}, \beta_{0}\right)-\hat{\theta}, \hat{\beta}\right\|$ is $O_{d}\left(\frac{1}{\sqrt{n}}\right)$ with probability at least $1-\delta$.

\section{PROJECTED GRADIENT DESCENT ANALYSIS}

We will use the following well known property of Projected Gradient Descent (Theorem 3.10 from [6]).

THEOREM 5.1. Let $f$ be $\alpha$-strongly convex and $\lambda$-smooth on compact set $\mathcal{X}$. Then projected gradient descent with stepsize $\eta=\frac{1}{\lambda}$ satisfies for $t \geq 0$

$$
\left\|\mathbf{x}_{t+1}-\mathbf{x}^{*}\right\|_{2}^{2} \leq e^{-\frac{\alpha t}{\lambda}}\left\|\mathbf{x}_{1}-\mathbf{x}^{*}\right\|_{2}^{2}
$$

Therefore, setting $R=\left\|\mathbf{x}_{1}-\mathbf{x}^{*}\right\|_{2}$ and by choosing $t=\frac{2 \lambda \ln \frac{R}{\epsilon}}{\alpha}$ it is guaranteed that $\left\|\mathbf{x}_{t+1}-\mathbf{x}^{*}\right\|_{2} \leq \epsilon$.

\subsection{Projected Gradient Descent for Logistic Regression}

We consider the function $\operatorname{LPL}(\theta, \beta)$ (log-pseudolikelihood as defined in Section 3) and we would like to approximate $(\hat{\theta}, \hat{\beta})$ within $\frac{1}{\sqrt{n}}$ in $\ell_{2}$ distance. The stepsize in Theorem 5.1 should be $\eta=\frac{1}{\sqrt{d \Theta^{2}+1}}$ by Remark 3.5.

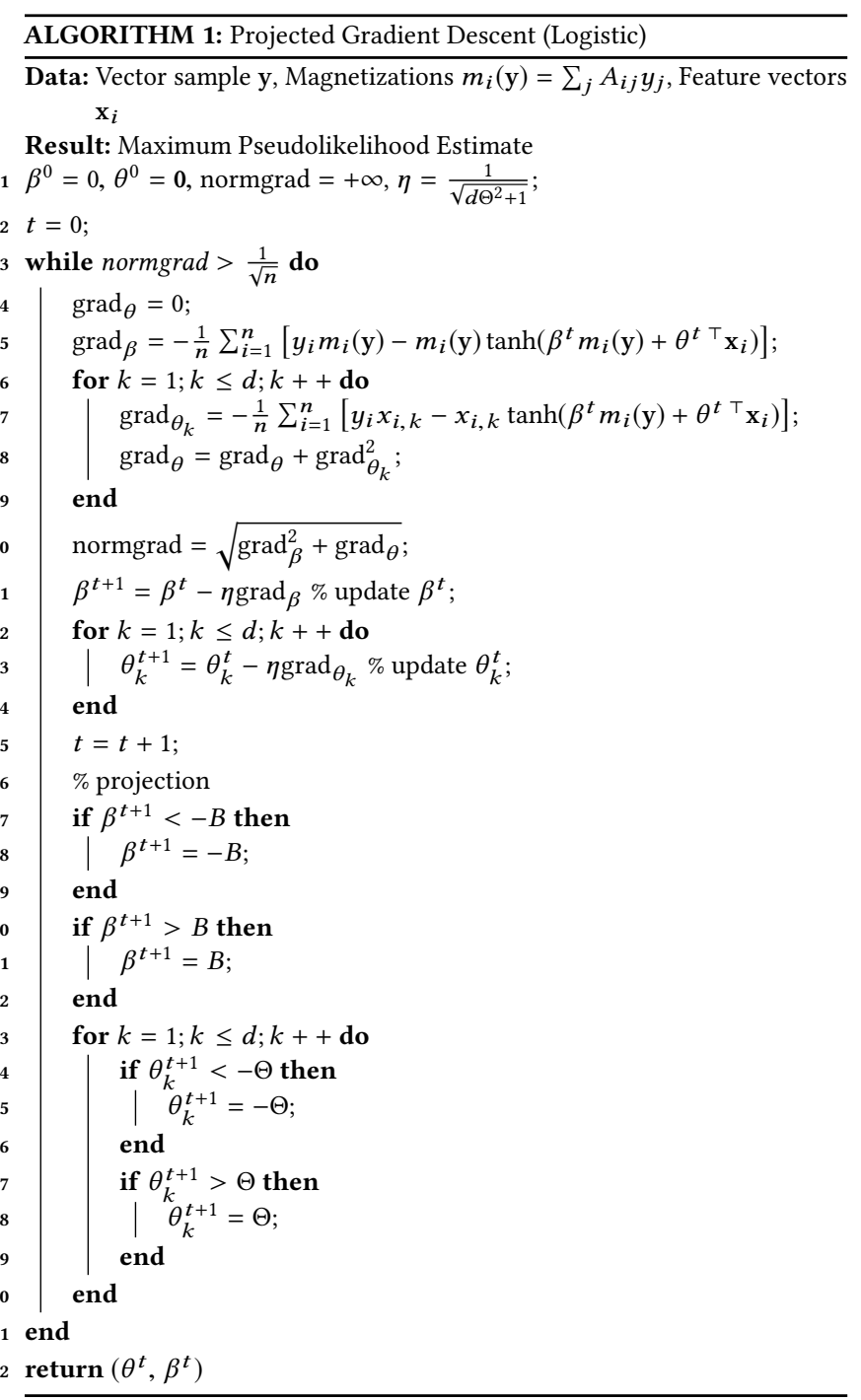

\section{ACKNOWLEDGMENTS}

Constantinos Daskalakis was supported by NSF awards CCF-1617730 and IIS-1741137, a Simons Investigator Award, a Google Faculty Research Award, and an MIT-IBM Watson AI Lab research grant. Nishanth Dikkala was supported by NSF awards CCF-1617730 and IIS-1741137. Ioannis Panageas was supported by SRG ISTD 2018 136. 


\section{REFERENCES}

[1] Alekh Agarwal and John C Duchi. 2013. The generalization ability of online algorithms for dependent data. IEEE Transactions on Information Theory 59, 1 (2013), 573-587.

[2] Marianne Bertrand, Erzo FP Luttmer, and Sendhil Mullainathan. 2000. Network effects and welfare cultures. The Quarterly fournal of Economics 115, 3 (2000), 1019-1055.

[3] Julian Besag. 1975. Statistical analysis of non-lattice data. The statistician (1975), 179-195.

[4] Bhaswar B Bhattacharya, Sumit Mukherjee, et al. 2018. Inference in Ising models Bernoulli 24, 1 (2018), 493-525.

[5] Yann Bramoullé, Habiba Djebbari, and Bernard Fortin. 2009. Identification of peer effects through social networks. Journal of econometrics 150, 1 (2009), 41-55.

[6] Sébastien Bubeck et al. 2015. Convex optimization: Algorithms and complexity. Foundations and Trends ${ }^{\circledR}$ in Machine Learning 8, 3-4 (2015), 231-357.

[7] Sourav Chatterjee. 2005. Concentration Inequalities with Exchangeable Pairs. Ph.D. Dissertation. Stanford University.

[8] Sourav Chatterjee. 2005. Concentration inequalities with exchangeable pairs ( $\mathrm{Ph}$ D. thesis). arXiv preprint math/0507526 (2005).

[9] Sourav Chatterjee et al. 2007. Estimation in spin glasses: A first step. The Annals of Statistics 35, 5 (2007), 1931-1946.

[10] Sourav Chatterjee and Amir Dembo. 2016. Nonlinear large deviations. Advances in Mathematics 299 (2016), 396-450.

[11] Nicholas A Christakis and James H Fowler. 2013. Social contagion theory: examining dynamic social networks and human behavior. Statistics in medicine 32,4 (2013), 556-577.

[12] Francis Comets and Basilis Gidas. 1991. Asymptotics of maximum likelihood estimators for the Curie-Weiss model. The Annals of Statistics (1991), 557-578.

[13] Constantinos Daskalakis, Nishanth Dikkala, and Siddhartha Jayanti. 2018. HOGWILD!-Gibbs can be PanAccurate. In Advances in Neural Information Processing Systems. 32-41.

[14] Constantinos Daskalakis, Nishanth Dikkala, and Gautam Kamath. 2017. Concentration of multilinear functions of the Ising model with applications to network data. In Advances in Neural Information Processing Systems. 12-23.

[15] Constantinos Daskalakis, Nishanth Dikkala, and Gautam Kamath. 2018. Testing ising models. In Proceedings of the Twenty-Ninth Annual ACM-SIAM Symposium on Discrete Algorithms. Society for Industrial and Applied Mathematics, 19892007.

[16] Constantinos Daskalakis, Elchanan Mossel, and Sébastien Roch. 2011. Evolutionary Trees and the Ising Model on the Bethe Lattice: A Proof of Steel's Conjecture. Probability Theory and Related Fields 149, 1 (2011), 149-189.

[17] Christopher De Sa, Kunle Olukotun, and Christopher Ré. 2016. Ensuring rapid mixing and low bias for asynchronous Gibbs sampling. In $7 M L R$ workshop and conference proceedings, Vol. 48. NIH Public Access, 1567.

[18] Esther Duflo and Emmanuel Saez. 2003. The role of information and social interactions in retirement plan decisions: Evidence from a randomized experiment. The Quarterly journal of economics 118, 3 (2003), 815-842.

[19] Glenn Ellison. 1993. Learning, Local Interaction, and Coordination. Econometrica 61, 5 (1993), 1047-1071.
[20] K. Fan and A.J. Hoffman. 1955. Some metric inequalities in the space of matrices. Proc. Amer.Math. Soc (1955), 111-116.

[21] Joseph Felsenstein. 2004. Inferring Phylogenies. Sinauer Associates Sunderland.

[22] Stuart Geman and Christine Graffigne. 1986. Markov Random Field Image Models and their Applications to Computer Vision. In Proceedings of the International Congress of Mathematicians. American Mathematical Society, 1496-1517.

[23] Promit Ghosal and Sumit Mukherjee. 2018. Joint estimation of parameters in Ising model. arXiv preprint arXiv:1801.06570 (2018).

[24] Edward L Glaeser, Bruce Sacerdote, and Jose A Scheinkman. 1996. Crime and social interactions. The Quarterly fournal of Economics 111, 2 (1996), 507-548.

[25] Linus Hamilton, Frederic Koehler, and Ankur Moitra. 2017. Information theoretic properties of Markov random fields, and their algorithmic applications. In Advances in Neural Information Processing Systems. 2463-2472.

[26] Kathleen Mullan Harris, National Longitudinal Study of Adolescent Health, et al. 2009. Waves I \& II, 1994-1996; Wave III, 2001-2002; Wave IV, 2007-2009 [machinereadable data file and documentation]. Chapel Hill, NC: Carolina Population Center, University of North Carolina at Chapel Hill 10 (2009).

[27] Ernst Ising. 1925. Beitrag zur Theorie des Ferromagnetismus. Zeitschrift für Physik A Hadrons and Nuclei 31, 1 (1925), 253-258.

[28] Adam Klivans and Raghu Meka. 2017. Learning graphical models using multiplicative weights. In 2017 IEEE 58th Annual Symposium on Foundations of Computer Science (FOCS). IEEE, 343-354.

[29] David A. Levin, Yuval Peres, and Elizabeth L. Wilmer. 2009. Markov Chains and Mixing Times. American Mathematical Society.

[30] Tianxi Li, Elizaveta Levina, and Ji Zhu. 2016. Prediction models for networklinked data. arXiv preprint arXiv:1602.01192 (2016)

[31] Charles F Manski. 1993. Identification of endogenous social effects: The reflection problem. The review of economic studies 60, 3 (1993), 531-542.

[32] Ron Meir. 2000. Nonparametric time series prediction through adaptive model selection. Machine learning 39, 1 (2000), 5-34

[33] Dharmendra S Modha and Elias Masry. 1996. Minimum complexity regression estimation with weakly dependent observations. IEEE Transactions on Information Theory 42, 6 (1996), 2133-2145.

[34] Mehryar Mohri and Afshin Rostamizadeh. 2010. Stability bounds for stationary $\varphi$-mixing and $\beta$-mixing processes. Journal of Machine Learning Research 11, Feb (2010), 789-814.

[35] Andrea Montanari and Amin Saberi. 2010. The Spread of Innovations in Social Networks. Proceedings of the National Academy of Sciences 107, 47 (2010), 2019620201.

[36] Bruce Sacerdote. 2001. Peer effects with random assignment: Results for Dartmouth roommates. The Quarterly journal of economics 116, 2 (2001), 681-704.

[37] D. Sherrington and S. Kirkpatrick. 1975. Solvable model of a spin-glass. Phys. Rev. Lett. 35 (1975)

[38] Ingo Steinwart and Andreas Christmann. 2009. Fast learning from non-iid observations. In Advances in neural information processing systems. 1768-1776.

[39] Justin G Trogdon, James Nonnemaker, and Joanne Pais. 2008. Peer effects in adolescent overweight. Fournal of health economics 27, 5 (2008), 1388-1399.

[40] Bin Zou, Luoqing Li, and Zongben Xu. 2009. The generalization performance of ERM algorithm with strongly mixing observations. Machine learning 75, 3 (2009), 275-295. 